\title{
What use is a clinical case definition for AIDS in Africa?
}

\author{
Charles F Gilks
}

In 1986, after a workshop was held in Bangui, Central African Republic, the World Health Organisation proposed a clinical case definition for identifying adults with AIDS. ${ }^{1}$ Previously the organisation had adopted the Centers for Disease Control case definition of AIDS for surveillance purposes. ${ }^{1}$ It was recognised, however, that this definition was not applicable in countries with limited diagnostic resources because it was based on establishing an aetiological diagnosis and needed, in most cases, a positive result on serological testing for HIV. The clinical case definition was therefore drawn up as a surveillance tool which used clinical signs and symptoms alone and did not require any confirmatory laboratory data. Various signs and symptoms were grouped into major and minor criteria to be used in a way thought most likely to identify adults with a probable underlying diagnosis of AIDS, once other causes of immunosuppression had been excluded.

The clinical case definition has become widely accepted and used in sub-Saharan Africa, either in the original form or with minor modifications. ${ }^{2}$ There has been no major evaluation or critical appraisal of its use, despite the fact that it was originally proposed as a provisional surveillance tool. It may be, for the reasons outlined below, that such a review is now necessary.

\section{An unworkable concept}

The underlying assumption behind the clinical case definition is that patients with AIDS (those with the specific indicator diseases listed in the Centers for Disease Control surveillance definition) can reliably be identified without any laboratory investigations or serological testing being performed. Diagnosis made on the basis of clinical presentation alone is inexact because of the limited and similar response of each organ system to widely differing pathogens and pathologies. Clinical surveillance does not, however, need to

Kenya Medical Research Institute, Nairobi, Kenya Charles F Gilks, MRCP, visiting scientist

Correspondence to: $\mathrm{Dr}$ Charles F Gilks, KEMRI/ Wellcome Trust Research Programme, PO Box 43640, Nairobi, Kenya.

BMf 1991;303:1189-90 be diagnostically exact as a specific cause does not have to be identified. It is enough that the presentations of most problems that define AIDS are recognised as such and that they are sufficiently distinct from all diseases that are not AIDS defining in patients who are positive for HIV antibody and from all illness those who are negative for HIV antibody. But in patients with features of underlying immunosuppression an indicator opportunistic lung infection, for example, can present as pulmonary tuberculosis or a partially treated pneumonia; persistent diarrhoea with weight loss can be associated with opportunistic as well as ordinary enteric parasites and bacteria; and so on. This presumably is why clinical surveillance has never been considered useful or reliable in countries with readily available diagnostic facilities.

Such difficulties are amplified where morbidity not associated with AIDS, caused by non-opportunistic bacteria and mycobacteria, is common. This is my experience in Nairobi. ${ }^{3.5}$ It can also be difficult to exclude reliably chronic disease in patients negative for HIV antibody with diseases such as tuberculosis, ${ }^{46}$ renal failure, and uncontrolled diabetes. ${ }^{7}$ The clinical case definition may therefore be unworkable as it lacks sufficient clinical accuracy.

\section{The wrong definition}

It is possible that the clinical case definition has been constructed wrongly. Many patients are defined as having AIDS because chronic cough is the minor sign which accompanies the two major features. This was found in $46 \%$ of one series ${ }^{8}$ and $52 \%$ of another. ${ }^{9}$ The main diagnosis in Africa with this presentation is pulmonary tuberculosis ${ }^{10}$ rather than an opportunistic infection such as pneumocystis pneumonia." Several groups have discussed the difficulty in differentiating reliably between cases of pulmonary tuberculosis in patients who are positive for HIV antibody and in those who are-negative for it, and some have suggested modifying the clinical case definition accordingly..$^{28}$ What has not been emphasised, however, is that pulmonary tuberculosis is not, ${ }^{12}$ nor ever has been, an AIDS defining condition.

If different ways are used to define a case it is a fundamental epidemiological principle that they must be standardised and identify the same conditions. ${ }^{13}$ It is surprising that such an important inconsistency exists between the clinical case definition and the Centers for Disease Control definitions for AIDS. In those countries where the incidence of tuberculosis is high and which are using the unmodified clinical case definition for surveillance substantial numbers of people reported as having AIDS may in fact not have AIDS.

\section{Incorrect validation}

After its introduction the clinical case definition was evaluated in several different studies. The standard against which the definition was validated was specific antibody to HIV, as suggested by the World Health $\begin{aligned} & \text { National surveillance of AIDS cases is not practical in poor African } \\ & \text { countries with inadequate registration systems }\end{aligned}$
Control definition of AIDS, ${ }^{12}$ which should be the true 
standard. In published papers which use HIV antibody status as the reference sensitivity has ranged from $52 \%$ to $59 \%$, specificity from $78 \%$ to $90 \%$, and positive predictive value from $50 \%$ to $74 \% .^{2814}$ The conclusions have been that the results support the widespread use of the clinical case definition for diagnosing AIDS in Africa. As the wrong standard has been used for validation, however, all that can be concluded is that the definition is specific but relatively insensitive at identifying seropositive patients. No conclusions can be drawn about the ability of the definition to identify people with AIDS unless the assumption is made that all HIV related disease in seropositive adults is AIDS. This is clearly not the case.

In Nairobi we have validated the clinical case definition against the Centers for Disease Control definition. Although the sensitivity (79\%) and specificity $(91 \%)$ were acceptable, the positive predictive value was only $30 \%$ (19/63 in 506 patients with a prevalence of $4 \cdot 8 \%$ for AIDS according to the Centers for Disease Control definition. ${ }^{15}$ With such a low positive predictive value it is difficult to see how such a test is of any value in surveillance for AIDS.

\section{Improper use}

Although the clinical case definition was proposed as a surveillance tool, its use seems to have been extended beyond this. Some doctors apply it or recommend it for individual patient management. ${ }^{14} 16$ By itself a diagnosis of AIDS is of little help unless the underlying indicator disease is known or strongly suspected. The clinical case definition contains no information about what the AIDS defining problem is likely to be for a given presentation or what is appropriate treatment. Labelling a patient as having AIDS can also result in negative consequences, especially if the person is in fact negative for HIV antibody. Other researchers have used the definition to diagnose individual patients as having AIDS so that they can then be compared with North American patients with AIDS, either in the serological response to HIV infection ${ }^{17}$ or in natural history studies. ${ }^{18}$ To compare patients so imprecisely labelled by the definition (even if this is backed up by serological results) with patients diagnosed by rigorous application of the Centers for Disease Control definition is problematic; in the light of what has been discussed above it may be invalid.

\section{A poor surveillance tool}

The clinical case definition may not be fulfilling its primary role as a useful surveillance tool. While counting cases of AIDS is an important way of monitoring the spread and the impact of the HIV epidemic in the USA, it does not necessarily follow that it is the right approach elsewhere. Vital registration systems do not exist or are fragmentary in many of the poorer African countries, so it is unlikely that national surveillance by using any clinical definition will work universally. There are alternative ways of obtaining more accurate data about the spread and impact of HIV in the community (for example, national or regional cluster sampling; anonymous testing of pregnant women and attenders of sexually transmitted diseases clinics; hospital surveillance of selected patient populations such as those admitted as medical emergencies, patients with pulmonary tuberculosis, and those who die when inpatients). Basing surveillance on cases of AIDS also ignores all clinical disease and death not associated with AIDS, even though this may be numerically more important

In Nairobi most of the HIV related morbidity seen is not AIDS defining, nor is it occurring in patients with a previous diagnosis of AIDS according to the Centers for Disease Control definition. . $^{3.5}$ One necropsy survey of adults in the Ivory Coast found that $16 \%$ had had AIDS (by clinical diagnosis) but that $38 \%$ had been positive for HIV antibody. ${ }^{19}$ Counting cases of AIDS is now probably not the most useful way of monitoring the HIV epidemic in Africa.

\section{Conclusions}

I have presented a case for reviewing the provisional clinical case definition. It might even be better to abandon the definition entirely. Africa has moved on tremendously in its efforts to combat the HIV epidemic since the clinical case definition was first proposed. Serological testing of a high quality is now available, to an extent perhaps not thought possible in 1986. Much more is now known about the epidemic and its clinical consequences. There seems little point in persisting with a provisional surveillance definition which is inherently unworkable and incorrect and which ignores the considerable changes that have taken place since its inception. Abandoning the clinical case definition may also reduce the undue emphasis currently placed on opportunistic infections and AIDS to the exclusion of other problems. ${ }^{20}$ Serious disease and premature death caused by HIV immunosuppression are important in Africa regardless of whether they fulfil a North American definition for AIDS or not. If i is considered worth while to continue with clinical surveillance then surely serious morbidity and mortality are what should be monitored.

The views and opinions expressed in this paper are those of the author and should not be construed as official or reflecting the views of the Kenya Medical Research Institute or the University of Oxford.

1 World Health Organisation. Acquired immunodeficiency syndrome. WHO CDC case definition for AIDS. Weekly Epidemiological Records 1986;61: 69-76.

2 Widi-Wirski R, Berkley S, Downing, R, Okwave S, Recine U, Mugerwa MD et al. Evaluation of the WHO clinical case definition for AIDS in Uganda. IAMA 1988:260:3286-9.

3 Gilks CF, Brindle RJ, Otieno LS, Simani P, Newnham RS, Bhatt SM, et al. Life-threatening bacteraemia in HIV-1 seropositive adults admitted to Life-threatening bacteraemia in HIV-1 seropositive

4 Gilks CF, Brindle RJ, Otieno LS, Simani P, Newnham RS, Bhatt SM, et al. Extrapulmonary and disseminated tuberculosis in HIV-1 seropositive patients presenting to the acute medical services in Nairobi. AIDS 990;4:981-5.

5 Gilks CF, Brindle RJ, Ojoo SA. Non-opportunistic bacterial infections in HIV seropositive adults in Nairobi. AIDS (in press).

6 Colebunders RL, Braun MM, Nzila N, Dikilu K, Muepu K, Ryder R. Evaluation of the WHO clinical case definition of AIDS among tuberculosi patients in Kinshasa, Zaire. F Infect Dis 1989;160:902-3.

7 Swai ABM, Lyimo PJM, Rutayuga F, McLarty DG. Diabetes mellitu misdiagnosed as AIDS. Lancet 1989;ii:976.

8 Colebunders R, Mann JM, Francis H, Bih K, Zaley L, Katonde N, et al. Evaluation of a clinical case definition of AIDS in Africa. Lance 1987;i:492-4.

Reeve PA. HIV infection in patients admitted to a general hospital in Malawi. BMF 1989;298:1567-8.

10 Harries AD. Tuberculosis and HIV infection in developing countries. Lancet $1990 ; 335: 387-90$

11 Lucas SB. Missing infections in AIDS. Trans $R$ Soc Trop Med Hyg 1990;84(suppl 1):34-8.

12 Centers for Disease Control. 1987 Revision of case definition for surveillance purposes. MMWR 1987;36(suppl 1):4-9S

13 Rose G, Barker DJP. What is a case? Dichotomy or continuum? BMY 1978;ii: $873-4$

14 De Cock KM, Colebunders RL, Francis H, Nzilambi N, Laga M, Ryder RW, et al. Evaluation of the WHO clinical case definition for AIDS in rural Zaire. AIDS 1988;2:219-21.

15 Gilkes CF, Brindle RJ, Otieno LS, Simani P, Newnham RS, Bhatt SH, et al. The presentation and outcome of HIV related disease in Nairobi. $Q \mathcal{F} M e d$ (in press).

16 Wabwire-Mangen F, Serwadda D, Sewankambo NK, Mugerwa RD, Shiff CJ Vlahor D, et al. Further experience with the WHO clinical case definition for AIDS in Uganda. AIDS 1989;3:462-3.

17 Brown C, Kline R, Atibu L, Francis H, Ryder R, Quinn TC. Prevalence of HIV-1 p24 antigenaemia in African and North American populations and correlation with clinical status. AIDS 1991:5:89-92.

18 Mann JM, Bila K, Colebunders RL Kalemba K, Khonde N, Bosenge N, etal. Natural history of HIV infection in Zaire. Lancet 1986;ii:707-9.

19 De Cock KM Barrere B, Diection in Zaire. Lancet 1986 ,ii. $B$. AIDS - the leading cause of adult death in the west African city of Abidjan, AIDS - the leading cause of adult death

20 Gilks $C F$, Ojoo $S A$. A practical approach to the clinical problems of the HIV infected adult in the tropics. Tropical Doctor 1991 (in press).

(Accepted 2l August 199] 